Mathematical Programming manuscript No.

(will be inserted by the editor)

François Margot

\title{
Pruning by Isomorphism in Branch-and-Cut
}

the date of receipt and acceptance should be inserted later

Abstract. The paper presents a branch-and-cut for solving $(0,1)$ integer linear programs having a large symmetry group. The group is used for pruning the enumeration tree and for generating cuts. The cuts are non-standard, cutting integer feasible solutions but leaving the optimal value of the problem unchanged. Pruning and cut generation are performed by backtracking procedures using a Schreier-Sims table for representing the group. Applications to hard set covering problems and to the generation of covering designs and error correcting codes are presented.

Key words. Branch-and-cut - isomorphism pruning - symmetry

\section{Introduction}

Let $\Pi^{n}$ be the set of all permutations of the ground set $I^{n}=\{1, \ldots, n\}$. A permutation in $\Pi^{n}$ is represented by an $n$-vector $\pi$, with $\pi[i]$ being the image of $i$ under $\pi$. If $v$ is an $n$-vector and $\pi \in \Pi^{n}$, let $w=\pi(v)$ denote the vector $w$ obtained by permuting the coordinates of $v$ according to $\pi$, i.e.

$$
w[\pi[i]]=v[i] \text { for all } i \in I^{n} .
$$

We consider an ILP problem of the form

$$
\begin{array}{cc}
\min & c^{T} \cdot x \\
\text { s.t. } & A x \geq b, \\
& x \in\{0,1\}^{n},
\end{array}
$$

where $A$ is an $m \times n$ matrix. For a permutation $\pi$ of the $n$ variables such that $\pi(c)=c$ and a permutation $\sigma$ of the $m$ rows of $A$ such that $\sigma(b)=b$, let $A(\pi, \sigma)$ be the matrix obtained from $A$ by permuting its columns according to $\pi$ and its rows according to $\sigma$. Let

$$
G=\{\pi \mid \text { there exists } \sigma \text { s.t. } A(\pi, \sigma)=A\} .
$$

François Margot: Department of Mathematics, University of Kentucky, Lexington, KY 405060027, e-mail: fmargot@ms.uky.edu 
Clearly, $G$ is a permutation group of $I^{n}$. Moreover, for $\pi \in G$, a point $\bar{x}$ is feasible (resp. optimal) for the linear relaxation of the ILP (1) if and only if $\pi(\bar{x})$ is feasible (resp. optimal) for that ILP. Hence, $G$ is a symmetry group of the feasible (and of the optimal) set of the ILP.

ILPs with large symmetry groups occur naturally when formulating classical problems in combinatorics, for example problems looking for a family of subsets of a given set $E$ with specified properties. In most cases, the elements in $E$ are indistinguishable and $G$ is a group with order at least $|E|$ !. The problem of scheduling jobs on $p$ parallel identical machines also yields ILPs with a natural symmetry group with at least $p$ ! elements. For relatively modest size problems, it turns out that the corresponding ILPs become very difficult (if not impossible) to solve by traditional branch-and-cut techniques. The trouble comes from the fact that many subproblems in the enumeration tree will be isomorphic, forcing a wasteful duplication of effort.

In this paper, we assume that an ILP together with its symmetry group $G$ is given. We show how to use $G$ in order to efficiently prune isomorphic subproblems and to help the search by generating isomorphism cuts (cutting integer feasible solutions, but leaving the value of the optimal solution unchanged). This isomorphism pruning is compatible with standard cut generation techniques (Gomory cuts, Lift-and-Project cuts, or specially designed cuts for the problem at hand). The price to pay for the pruning is that the branching variable can no longer be chosen arbitrarily. We also assume that the reader is familiar with the branchand-cut procedure, as excellent introductions can be found in [33], [37], [38].

While isomorphism rejection in backtracking searches has been used in many applications [4], [6], [7], [14], [17], [20], [21], [22], [23], [29], [34], [35], it is not commonly used in a branch-and-cut context. In most instances, the symmetry group $G$ is not assumed to be known and the backtracking search has the additional task to produce it. The originality of the proposed approach resides essentially in (i) the possibility of generating isomorphism cuts (that will be shown to be efficient for the covering design problem), and (ii) the development of algorithms for computing orbits and stabilizers of sets under a group, taking advantage of the type of stabilizers and points in the queries needed by the branch-and-cut.

Section 2 describes the pruning algorithm, and Section 3 presents basic data structures and algorithms for group operations. Section 4 describes the restrictions that can be put on queries for orbits and stabilizers generated during the branch-and-cut. Section 5 introduces the isomorphism cuts. Finally, Section 6 presents results on three applications: set covering problems, covering designs and error correcting codes.

We close this section with two basic definitions and some notation: 
Let $S \subseteq I^{n}$. To simplify the notation, we use a set $S$ and its characteristic vector interchangeably.

The orbit of $S$ under $G$ is

$$
\operatorname{orb}(S, G)=\left\{S^{\prime} \subseteq I^{n} \mid S^{\prime}=g(S) \text { for } g \in G\right\} .
$$

The stabilizer of $S$ in $G$ is the subgroup of $G$ given by:

$$
\operatorname{stab}(S, G)=\{g \in G \mid g(S)=S\} .
$$

For $1 \leq a \leq b \leq n$, we write $v[a . . b]$ to denote the entries $\{v[a], v[a+1], \ldots, v[b]\}$ of $v$ as an unordered set.

If $g_{1}, \ldots, g_{k}$ are $k$ permutations of $I^{n}$, the permutation $g=g_{1} \cdot \ldots \cdot g_{k}$ is obtained by applying the permutations from right to left, i.e $g(v)=g_{1}\left(g_{2}\left(\ldots\left(g_{k}(v)\right) \ldots\right)\right)$ for any $n$-vector $v$.

\section{Isomorphism Test, Pruning, and Fixing}

The proposed branch-and-cut will branch by fixing the value of one variable $x_{j}$ to 0 or 1 . Since the ILP (1) has a large automorphism group $G$, it is very likely that several nodes in the enumeration tree will correspond to isomorphic problems. Obviously, solving one of these isomorphic problems and pruning the others would result in huge savings. One important goal is to do so without having to keep in memory the list of all non-isomorphic subproblems encountered since the start of the algorithm. One way to achieve this is to define, for each isomorphism class of subproblems, one particular subproblem (called the representative of the class) that will be solved. Given a subproblem, we then need only to be able to decide if it is a representative or not. If it is not, we can prune the corresponding node of the branch-and-cut. Some care must be taken to ensure that the representative subproblems form a subtree of the branch-and-cut tree including the root. The general approach of isomorphism free generation of combinatorial structures based on representatives was studied by Read [34]. A general theory for isomorphism free generation, developed by McKay, can be found in [29].

We distinguish fixed variables from set variables: branching decisions fix variables whereas logical implications or other tests set variables. We will use only one operation, called 0-setting, to set variables to 0 . It is important to realize that the results below may not hold if additional setting operations are used. Let $a$ be a node of the branch-and-cut enumeration tree. Let $F_{1}^{a}\left(\right.$ resp. $\left.F_{0}^{a}\right)$ be the set of indices of variables fixed to 1 (resp. fixed to 0 ) at $a$. Let $F S_{0}^{a}$ be the set of indices of variables fixed or set to 0 at $a$. Let $F^{a}$ be the set of indices of variables that 
are not in $F_{1}^{a} \cup F S_{0}^{a}$, variables also called free at $a$. Let $b$ be another node and let $F_{1}^{b}, F_{0}^{b}$ be the corresponding set of indices of variables at $b$. The subproblems associated with nodes $a$ and $b$ of the branch-and-cut are isomorphic if there exists a permutation $g \in G$, such that $g\left(F_{i}^{a}\right)=F_{i}^{b}$ for $i=0,1$.

Unfortunately, using this isomorphism test to identify subproblems that can be pruned during the branch-and-cut would require the storage of a maximal set of non-isomorphic subproblems generated so far in the enumeration. Moreover, the computation needed to determine if $g$ exists is not trivial and would be required for many pairs of subproblems. Using the definition of a representative, we can use a slight relaxation of the isomorphism test that turns out to be practical. The price to pay for the simplification is that we will no longer be free to branch on any variable of the ILP: at node $a$, the branching variable will have to be $x_{f}$ where $f$ is the minimum index in $F^{a}$ (even if the value of $x_{f}$ in the current solution of the LP relaxation is 0 or 1 ). The variable $x_{f}$ is called the branching variable at $a$. This branching strategy is called minimum index branching (MIB). The enumeration tree generated by a branch-and-bound $\mathcal{B}$ using the LP relaxation of (1) to prune only infeasible subproblems is called the full enumeration tree of $\mathcal{B}$. By convention, the full enumeration tree only contains nodes that are not pruned.

Let $S \neq T$ be two subsets of $I^{n}$. Let $S_{[j]}$ (resp. $T_{[j]}$ ) denote the $j$ th smallest element in $S$ (resp. $T$ ). Then $S$ is lexicographically smaller than $T$ if there exists $k \in\{1, \ldots,|S|\}$ with $k<|T|$ such that $S_{[j]}=T_{[j]}$ for $j=1, \ldots, k$ and either $|S|=k$ or $S_{[k+1]}<T_{[k+1]}$. We write $S \preceq T$ if $S$ is equal to $T$ or if $S$ is lexicographically smaller than $T$.

A set $S \subseteq I^{n}$ is a representative if $S$ is lexicographically minimum among the sets in its orbit under $G$, i.e.

$$
S \preceq g(S) \forall g \in G .
$$

The following property is crucial for the validity of the pruning:

Lemma 1. Let $S \subseteq I^{n}$ be a representative under $G$. Let $S^{\prime}:=S-v$ with $v=\max \{w \in S\}$. Then $S^{\prime}$ is also a representative.

Proof. If $S^{\prime}$ is not a representative, then there exists $g \in G$ such that $g\left(S^{\prime}\right) \preceq S^{\prime}$. Then $g(S) \preceq S$, a contradiction.

Consider the following isomorphism pruning (IP) to be applied on nodes of the enumeration tree of a branch-and-cut: if $F_{1}^{a}$ is not a representative, then prune node $a$.

Lemma 2. Let $\tau$ be the full enumeration tree of a branch-and-cut $\mathcal{B}$ using MIB. Let $S$ be the nodes in $\tau$ that are not pruned by IP. Then 
(i) $S$ induces a subtree of $\tau$ containing the root of $\tau$;

(ii) The branch-and-cut $\mathcal{B}^{\prime}$ obtained by adding IP to $\mathcal{B}$ returns the same optimal value as $\mathcal{B}$.

Proof. (i): Let $a \in S$ and let $b \in \tau$ on the path between the root and $a$ in $\tau$. Then $F_{1}^{a}$ is a representative and, by the choice of branching strategy, $F_{1}^{b}$ is the set of the $\left|F_{1}^{b}\right|$ smallest entries in $F_{1}^{a}$. By Lemma $1, F_{1}^{b}$ is a representative, i.e. $b \in S$.

(ii): Let $a$ be a node of $\tau$ for which $F_{1}^{a}$ is an optimal solution to ILP (1). Then the representative of the orbit of $F_{1}^{a}$ under $G$ is a set $F^{*}$, and thus there is a node $b \in S$ with $F_{1}^{b}=F^{*}$. By (i), the full enumeration tree of $\mathcal{B}^{\prime}$ is the subtree induced by $S$ in $\tau$. This implies that $\mathcal{B}^{\prime}$ will process node $b$ at some point, yielding the same optimal value as the one returned by $\mathcal{B}$.

When solving a subproblem $a$, it is sometimes possible to identify variables that may be set to 0 without affecting the optimal solution returned by a branchand-cut using MIB and IP. Consider the following operations:

(i) Let $b$ be the father of $a$ in the enumeration tree and let $x_{f}$ be the branching variable at $b$. If $a$ is the son of $b$ where $x_{f}$ is fixed to 0 then set to 0 all free variables in $\operatorname{orb}\left(f, \operatorname{stab}\left(F_{1}^{a}, G\right)\right)$.

(ii) Let $f=\min \left\{r \in F^{a}\right\}$. If $F_{1}^{a} \cup f$ is not a representative, then set to 0 all free variables in $\operatorname{orb}\left(f, \operatorname{stab}\left(F_{1}^{a}, G\right)\right)$.

Applying these operations (repeatedly for (ii) if possible, i.e. until no free variable exists or until $F_{1}^{a} \cup f$ is a representative) is called performing a 0 -setting. The output of the 0 -setting is the value $f$ in (ii) for which $F_{1}^{a} \cup f$ is a representative, or $n+1$ is no such $f$ exists.

Remark 1. Trivially, the variables set to 0 during a 0 -setting at node $a$ all have a larger index than the maximum index $M$ in $F_{1}^{a}$, since each variable in $F^{a}$ has a larger index than $M$.

Lemma 3. Consider a branch-and-cut $\mathcal{B}$ using $M I B$ and IP, and let $\mathcal{B}^{\prime}$ be the branch-and-cut obtained by adding 0-setting in $\mathcal{B}$. Then the optimal values returned by $\mathcal{B}$ and $\mathcal{B}^{\prime}$ are equal.

Proof. Let $a$ be a node of the full enumeration tree $\tau$ of $\mathcal{B}$ for which $F_{1}^{a}$ is an optimal solution to ILP (1). Then $F_{1}^{a}$ is a representative. Assume that no node $b$ in the full enumeration tree $\tau^{\prime}$ of $\mathcal{B}^{\prime}$ has $F_{1}^{b}=F_{1}^{a}$. Hence there exists a node $c \in \tau^{\prime}$ such that $F_{1}^{c}$ contains the $\left|F_{1}^{c}\right|$ smallest indices in $F_{1}^{a}$ and, during the 0 -setting at $c$, one of the variables in $F_{1}^{a}-F_{1}^{c}$ is set to 0 . Assuming that $c$ is 
chosen as close as possible to the root, we then have $j \in \operatorname{orb}\left(f, \operatorname{stab}\left(F_{1}^{c}, G\right)\right)$ for some $j \in F_{1}^{a}-F_{1}^{c}$ and $f \in F_{0}^{c}$ with

$$
\max \left\{r \in F_{1}^{c}\right\}<f<m:=\min \left\{r \in\left(F_{1}^{a}-F_{1}^{c}\right)\right\} \leq j .
$$

The first inequality comes from Remark 1 and the second one from the fact that $F_{1}^{c} \cup m$ is a representative: If $m$ is set to 0 during the 0 -setting at $c$, then it is from a $f<m$, and if $m$ is not set to 0 , then all $f$ considered during the 0 -setting are smaller than $m$.

Thus there exists $g \in \operatorname{stab}\left(F_{1}^{c}, G\right)$ such that $g[j]=f$. Then $g\left(F_{1}^{c} \cup j\right)=F_{1}^{c} \cup$ $f$ which is lexicographically smaller than $F_{1}^{c} \cup m$, proving that $F_{1}^{a}$ is not a representative as $F_{1}^{c} \cup j \subseteq F_{1}^{a}$, a contradiction.

It remains to show how to compute $\operatorname{orb}\left(f, \operatorname{stab}\left(F_{1}^{a}, G\right)\right)$ and how to test if a set is a representative or not. This will be covered in Section 4. In the remainder of the paper, the branch-and-cut is assumed to use MIB, IP and 0-setting. The operations performed at node $a$ in the enumeration tree are thus:

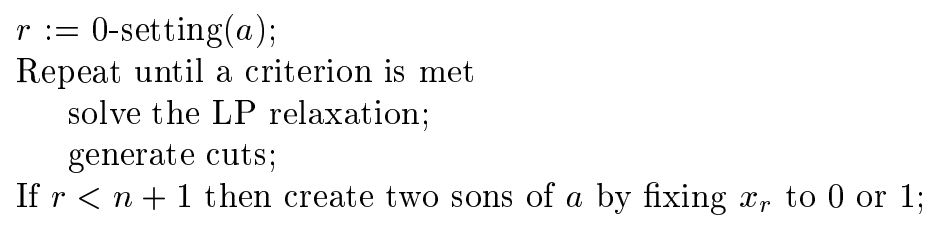

\section{Group Representation and Basic Algorithms}

Essentially two options are available to represent a permutation group $G$ : the explicit representation or a representation by generators. The explicit representation simply stores in a list each permutation in $G$. A representation by generators stores only a subset $\left\{g_{1}, \ldots, g_{k}\right\}$ of the permutations in $G$, with the property that any permutation in $G$ can be written as a product of permutations in the subset. If $|G|$ is small, the explicit representation might work well, but in most cases of interest a representation by generators is required. The operations of interest listed above are also, usually, faster with the representation by generators.

We use the Schreier-Sims representation of $G$ (also called strong generators) [4], [5], [6], [7], [16], [20], [21], [22]. (A good introduction can be found in [20].) Let

$$
\begin{aligned}
& G_{0}=G \\
& G_{1}=\left\{g \in G_{0} \mid g[1]=1\right\} \\
& G_{2}=\left\{g \in G_{1} \mid g[2]=2\right\} \\
& \ldots \\
& G_{n}=\left\{g \in G_{n-1} \mid g[n]=n\right\} .
\end{aligned}
$$


$G_{1}$ is simply the stabilizer of 1 in $G$, and $G_{i}$ is the stabilizer of $i$ in $G_{i-1}$. It follows that $G_{0}, G_{1}, \ldots, G_{n}$ are nested subgroups of $G$.

Example 1. Consider the group $G$ of symmetries of the $2 \times 2$ square, with one variable associated with each square as indicated below:

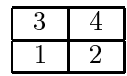

$G=G_{0}$ comprises 8 permutations: the identity $I=[1,2,3,4]^{T}$, three rotations $R_{90}=[2,4,1,3]^{T}, R_{180}=[4,3,2,1]^{T}, R_{270}=[3,1,4,2]^{T}$, the vertical symmetry $V=[2,1,4,3]^{T}$, the horizontal symmetry $H=[3,4,1,2]^{T}$, the symmetry along the main diagonal $M=[4,2,3,1]^{T}$, and the symmetry along the other diagonal $D=[1,3,2,4]^{T}$. Then $G_{1}=\{I, D\}$, and $G_{2}=G_{3}=G_{4}=\{I\}$.

For $k=1, \ldots, n$, let $\operatorname{orb}\left(k, G_{k-1}\right)=\left\{j_{1}, \ldots, j_{p}\right\}$ be the orbit of $k$ under $G_{k-1}$. Then for each $1 \leq i \leq p$, let $h_{k, j_{i}}$ be a permutation in $G_{k-1}$ sending $k$ on $j_{i}$, i.e. $h_{k, j_{i}}[k]=j_{i}$. Let $U_{k}=\left\{h_{k, j_{1}}, \ldots, h_{k, j_{p}}\right\}$. Note that $U_{k}$ is never empty as $\operatorname{orb}\left(k, G_{k-1}\right)$ always contains $k$.

Arrange the permutations in the sets $U_{k}, k=1, \ldots, n$ in an $n \times n$ table $T$, with

$$
T_{k, j}=\left\{\begin{aligned}
h_{k, j} & \text { if } j \in \operatorname{orb}\left(k, G_{k-1}\right), \\
\emptyset & \text { otherwise. }
\end{aligned}\right.
$$

The table $T$ is called the Schreier-Sims representation of $G$. This table is not uniquely defined, as there is usually a choice for the permutations included in the sets $U_{k}$. However, the general shape of the table (i.e. which entries are empty or not) is fixed.

Example 2. For the group $G$ of Example 3.1, we have $\operatorname{orb}\left(1, G_{0}\right)=\{1,2,3,4\}$, $\operatorname{orb}\left(2, G_{1}\right)=\{2,3\} \operatorname{orb}\left(3, G_{2}\right)=\{3\}$, and $\operatorname{orb}\left(4, G_{3}\right)=\{4\}$. A Schreier-Sims representation of $G$ is then:

\begin{tabular}{c|cccc} 
& 1 & 2 & 3 & 4 \\
\hline 1 & $I$ & $V$ & $H$ & $R_{180}$ \\
2 & & $I$ & $D$ & \\
3 & & & $I$ & \\
4 & & & & $I$
\end{tabular}

Remark 2. It is more efficient to implement the table as a vector of ordered lists instead of as a 2-dimensional table, as most entries in the table are usually 
empty. However, algorithms are simpler to describe and understand for the 2dimensional table. The actual implementation uses a vector of ordered lists.

Remark 3. The most interesting property of this representation of $G$ is that each $g \in G$ can be uniquely written as

$$
g=g_{1} \cdot g_{2} \cdots \cdot g_{n}
$$

with $g_{i} \in U_{i}$ for $i=1, \ldots n$. Hence the permutations in the table form a set of generators of $G$. It is called a strong set of generators, since the equation (3) shows that $g \in G$ can be expressed as a product of at most $n$ permutations in the sets.

Given a permutation $g \in G$, it is easy to find the $n$ permutations $g_{1}, \ldots, g_{n}$ of equation (3): the permutations $g_{2}, \ldots, g_{n}$ all stabilize point 1 , forcing $g_{1}$ to be $T[1, g[1]]$. Then, as $g_{3}, \ldots, g_{n}$ all stabilize point 2 , we must have $\left(g_{1} \cdot g_{2}\right)[2]=g[2]$, i.e. $g_{2}[2]=\left(g_{1}^{-1} \cdot g\right)[2]$ and thus $g_{2}=T\left[2,\left(g_{1}^{-1} \cdot g\right)[2]\right]$. A similar reasoning yields $g_{3}, \ldots, g_{n}$.

It is possible to make a small generalization of the presentation by ordering the points of the ground set in an arbitrary order $\beta$, called the base of the table. In that case, the subgroups $G(\beta)_{k}$ for $k=1, \ldots, n$ are defined as the stabilizer of $\beta[k]$ in $G(\beta)_{k-1}$, with $G(\beta)_{0}=G$. The corresponding table is denoted by $T(\beta)$. Row $k$ of $T(\beta)$ corresponds to the element $k, U(\beta)_{k}$ is the set of non-empty entries in row $k$ of $T(\beta)$ and $J(\beta)_{k}$ denotes the set of indices $\left\{j \in I^{n} \mid T(\beta)[k, j] \neq \emptyset\right\}$, also called the basic orbit of $k$ in $T$, following the terminology of [22]. When the base $\beta$ is fixed, we sometimes drop the qualifier $(\beta)$ in these symbols, but from now on each table $T$ is defined with respect to a base.

Let an identity row be a row $\beta[i]$ in table $T(\beta)$ such that the only non-empty entry in that row is entry $T[\beta[i], \beta[i]]$ which is the identity permutation.

Remark 4. For any $k \in\{1, \ldots, n\}$, replacing rows $\beta[1], \ldots, \beta[k-1]$ in $T(\beta)$ by identity rows yields a Schreier-Sims representation of $G(\beta)_{k-1}$. Hence the permutations on rows $\beta[k], \ldots, \beta[n]$ of $T(\beta)$ form a set of generators of $G(\beta)_{k-1}$.

Two natural questions arise: how can we create the table $T(\beta)$, knowing the group $G$ either explicitly or by a family of generators, and how can we change the base $\beta$ of the representation? Algorithms for performing these operations can be found in [4], [6], [7], [16], [20], [21], [22]. The implemented algorithm, build(), to create the table is closest to [20] and uses two other routines, test() and enter(). The parameter first of these procedures always has value 1 and could thus be removed. However, the base change algorithm down() given below calls enter() with first $>1$. The algorithms given in this section are not the 
most efficient in terms of worst case complexity or space requirements, but their simplicity and satisfactory empirical efficiency motivate their selection.

test $(T, \beta, p$, first $)$

$/^{*}$ Returns the smallest $i$ such that the row $T[\beta[i]]$ is modified if permutation $p$ is added to the generators of the group represented by $T(\beta)$ or returns $(n+1)$ if $T(\beta)$ is not changed; $p$ is passed by reference. */

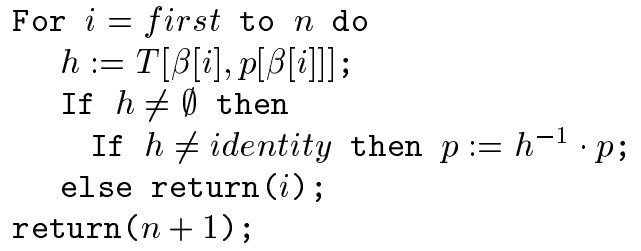

enter $(T, \beta, p$, first $)$

$/^{*}$ Add permutation $p$ to the generators of the group represented by $T(\beta) ; T$ is passed by reference. */

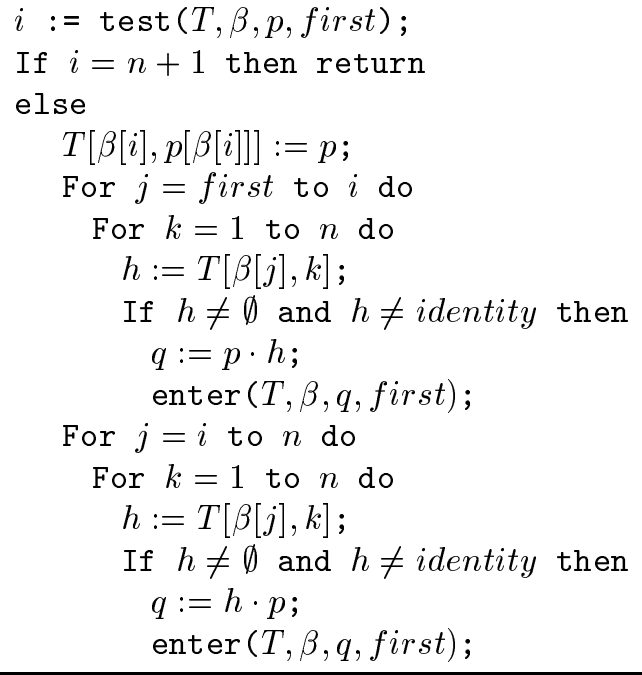

$\operatorname{build}(T, \beta, \mathcal{P})$

* $^{*}$ Build the table $T(\beta)$ for the group generated by the permutations in $\mathcal{P}$; $T$ is passed by reference. $* /$

Set all rows of $T(\beta)$ to identity rows;

For each $p \in \mathcal{P}$ do enter $(T, \beta, p, 1)$; 
Proposition 1. The algorithms test(), enter() and build() are correct.

Proof. As first $=1$ in the call enter $(n, T, \beta, p$, first) in build(), we get essentially the algorithms Test2(), Enter2() and Gen() of [20] (see also Algorithm 2 and 3 in Chapter II of [16]) where the proof of correctness can be found (Theorem 6.8 in [20]).

Remark 5. The complexity of one call to test() is in $O\left(n^{2}\right)$. The number of operations in one call to enter() is bounded as follows: $O\left(n^{2}\right)$ for one call to test(), and, if test() does not return $n+1, O\left(n^{2}\right)$ operations to run across the table plus, for each non-empty entry in the table $O(n)$ operations and one recursive call. Each time test() returns a number other than $n+1$, one additional entry in the table is filled. Thus test() returns a number other than $n+1$ at most $O\left(n^{2}\right)$ times and the total number of recursive calls to enter() is in $O\left(n^{4}\right)$. It follows that the complexity of enter() is in $O\left(n^{6}\right)$. A similar analysis shows that the complexity of build() is in $O\left(n^{6}+n^{2} \cdot|\mathcal{P}|\right)$. Faster algorithms for computing a representation of a group exist [2], [18], [36]. The complexity of the algorithm of Jerrum [18] is in $O\left(n^{5}+n^{2}|\mathcal{P}|\right)$ and the one of Babai et al. [2] is in $O\left(n^{4} \log ^{c} n+n^{2}|\mathcal{P}|\right)$ where $c$ is a constant. Since we might assume that the permutation group is given by a set of strong generators, the speed of the algorithm for finding the representation of the group is not particularly relevant to this work. However, the fact that the representations found by these algorithms require $O\left(n^{2}\right)$ space instead of $O\left(n^{3}\right)$ for the above algorithm could be of interest for applications where the Schreier-Sims table has close to $n^{2} / 2$ non-empty entries.

Given a table $T(\beta)$, a simple algorithm to change the base to $\beta^{\prime}$ is to use build $\left(n, T^{\prime}, \beta^{\prime}, \mathcal{P}\right)$ where $T^{\prime}$ is a new table and $\mathcal{P}$ is the set of non-empty entries in $T$. As the non-empty entries in $T$ form a set of generators of the group, the resulting table $T^{\prime}\left(\beta^{\prime}\right)$ is the wanted representation of the group. However, this procedure does not take advantage of the similarities between $T$ and $T^{\prime}$ when $\beta$ and $\beta^{\prime}$ are almost identical, as is the case for the base changes needed during the branch-and-cut: let $T(\beta)$ be the table at a node of the enumeration tree. As we will see in Section 4 , the base $\beta^{\prime}$ for any of its sons can be obtained through a few applications of the following operation (called downing of a point $v$ ): assume that $v=\beta[r]$ and let $r \leq s \leq n$. Let $\beta^{\prime}$ be the permutation obtained from $\beta$ by moving the entry $v$ to position $s$ of $\beta^{\prime}$, keeping the other entries in the same order as in $\beta$. The algorithm down() computes the table $T\left(\beta^{\prime}\right)$ efficiently. It is similar to an algorithm in [7] used to swap two adjacent entries in $\beta$. 


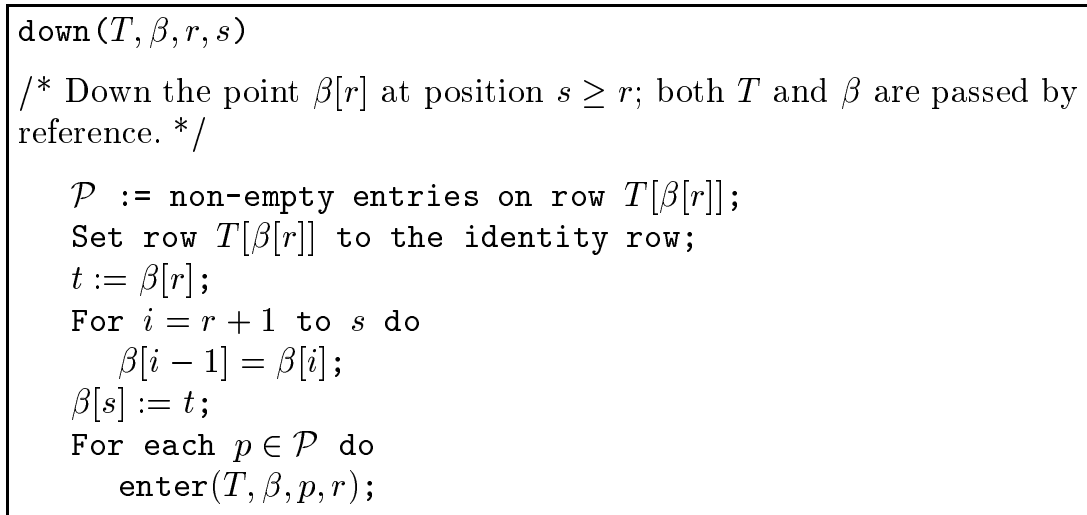

Proposition 2. The algorithm down() is correct.

Proof. Let $T^{\prime}$ and $\beta^{\prime}$ be the table and the base obtained after the call to down(). Let $T^{r}$ be the table obtained from $T$ by replacing rows $\beta[1 . . r]$ by identity rows. Let $G_{0}=G$ and let $G_{i}$ be the subgroup of all permutations in $G$ stabilizing each of the points $\beta[1 . . i]$ for $i=1, \ldots, n$ (c.f. $(2))$. Then $T^{r}(\beta)$ is a representation of $G_{r}$, as mentioned in Remark 4, and $T^{r}\left(\beta^{\prime}\right)$ is also a representation of $G_{r}$ as the only difference between the two representations is the position of an identity row. Notice that all calls to enter $\left(T, \beta^{\prime}, p\right.$, first $)$ are done with parameter first $=r$, and that the same calls and operations would be performed by calling enter $\left(T^{r}, \beta^{\prime}, p, 1\right)$. The latter returns a table representing the group obtained from $G_{r}$ by adding $p$ to the list of its generators. It follows that after entering all permutations in $\mathcal{P}$, the resulting table $T^{r^{\prime}}\left(\beta^{\prime}\right)$ is a description of $G_{r-1}$. Since rows $\beta^{\prime}[r . . n]$ of $T^{r \prime}$ and $T^{\prime}$ are identical, rows $\beta^{\prime}[r . . n]$ of $T^{\prime}$ are correct. Rows $\beta^{\prime}[1 . . r-1]$ of $T^{\prime}$ are identical to rows $\beta[1 . . r-1]$ of $T$ and this is also correct as the basic orbits of $\beta^{\prime}[i]=\beta[i]$ in $G_{i-1}$ for $i=1, \ldots, r-1$ are unchanged.

Remark 6. A crude analysis similar to the one in Remark 5 gives that the worst case complexity of down() is in $O\left(n^{3}+n^{4} \cdot k\right)$, where $k$ is the number of entries added to the table by the calls to enter(). A similar algorithm for performing the inverse of downing a point (an operation called a cyclic shift in [3]) has worst case complexity in $O\left(n^{3}\right)$. It is not obvious if the same ideas would extend or not to the case of downing a point. However, as $O(n)$ applications of the cyclic shift algorithm are enough to down a point, it is possible to down a point in $O\left(n^{4}\right)$. As down() is fast enough on the applications given in Section 6 and as the algorithm for the cyclic shift is much more elaborate, improvements for down() have not been explored.

An algorithm with worst case complexity in $O\left(n^{6}\right)$ or even $O\left(n^{4}\right)$ might seem impractical for values of $n \geq 100$. It turns out that the complexity bounds 
given in Remarks 5 and 6 are very pessimistic. The amount of time spent in the four algorithms described in this section during the branch-and-cut stays well below $5 \%$ of the total cpu time in typical applications. For example, for the covering designs application described in Section 6, $n=252$, the group has order $10 !=3,628,800$, but the number of entries in the table is, on average, 550 . Moreover, the distribution of the entries in the table is heavily biased towards the rows corresponding to the first entries in the base: typically, the cardinality of the basic orbits are: $\left|U_{\beta[0]}\right|=252,\left|U_{\beta[1]}\right|=25,\left|U_{\beta[2]}\right|=4,\left|U_{\beta[3]}\right|=3,\left|U_{\beta[4]}\right|=2$, $\left|U_{\beta[5]}\right|=1,\left|U_{\beta[6]}\right|=4$ and only two other basic orbits have cardinality larger than 1 (namely 3 and 2). This motivates the use of the parameter first in the algorithms test () and enter(): when first $>1$ (i.e. except at nodes of the branchand-cut enumeration tree where no variable is fixed to 1), 251 of the 295 entries of the table that are not identity permutations are ignored when looping through the table in enter(). Since each permutation considered in the loop involves a multiplication of two permutations and a call to test() (at a cost of $\Omega(n)$ ), this saves a substantial amount of work. This is of course an empirical observation. The unsophisticated worst case complexity analysis given above is unaffected. In any case, if the algorithms test(), enter() and down() are not satisfactory in term of space requirements or in execution speed for a particular application, it is possible to replace them by algorithms from [2], [3], [18] based on the compressed data structure of [18].

\section{Orbits, Stabilizers and Representatives}

We are interested in performing the following operations that were mentioned in Section 2: Computing the orbit of a point in the stabilizer of a set and deciding if a set is lexicographically minimum in its orbit under $G$.

For the former, if the stabilizer $G^{\prime}$ was given by a Schreier-Sims table, it would of course be possible to make a change of basis so that $v$ becomes the first entry of the basis, since then non-empty entries in row $v$ of the table will be the orbit of $v$ under $G^{\prime}$. In our particular case, however, $G^{\prime}$ is given implicitly and building the table for $G^{\prime}$ would be quite expensive. (Finding generators of the stabilizer of a set under $G$ is at least as hard as testing if two graphs are isomorphic [16], [24].) A faster algorithm can be found in [5], [16] but it also relies on a Schreier-Sims description of $G^{\prime}$.

We thus devised a backtracking algorithm for computing the orbit of a single point in the stabilizer of a set in $G$. It takes advantage of the fact that we might assume that the basis $\beta$ of the group at node $a$ of the enumeration tree has the following structure: variables fixed to 1 at $a$ (i.e. $F_{1}^{a}$ ) come first in $\beta$, then the free variables $\left(F^{a}\right)$, and then the variables fixed or set to 0 at $a\left(F S_{0}^{a}\right)$.

The data structure associated with group $G$ at node $a$ of the branch-and-cut is the following: 
table: $T$

integer: fixed_one base: $\beta$

vector: part_zero .

The table $T$ is just a Schreier-Sims representation of the group with base $\beta$. The variable fixed_one gives the number of variables in $F_{1}^{a}$ and

$$
F_{1}^{a}=\beta[1 . \text { fixed_one }] \quad \text { with } \quad \beta[1]<\cdots<\beta[\text { fixed_one }] .
$$

The vector part_zero is used to store information about variables fixed or set to 0 . For ind $=1, \ldots$,fixed_one, $\beta[$ part_zero $[i n d] . . n]$ are the variables that have been fixed or set to 0 before $\beta[i n d]$ was fixed to 1 . For ind $=$ fixed_one +1 , $\beta[$ part_zero $[i n d] . . n]=F S_{0}^{a}$, i.e. all the variables currently fixed or set to 0 at $a$. The remaining variables (the free ones) appear in $\beta$ in increasing order of their index, after variables in $F_{1}^{a}$ and before variables in $F_{0}^{a}$. Note that this structure of $\beta$ is easy to maintain throughout the branch-and-cut: when the 0 -setting is performed (or a variable is fixed to 0 by branching), free variables in a set $U$ are set to 0 . To update the table, simply use down(), moving one by one the variables in $U$. When a variable is fixed to 1 by branching, it is always the free variable with smallest index, and the basis (and thus the table) remains the same.

In this section, we consider algorithms for solving questions related to a single node $a$ of the branch-and-cut. To avoid heavy notations, the table associated with $a$ is denoted by $T$, instead of the more precise $a \rightarrow T$. The same remark applies to the three other fields of the data structure associated with $a$.

The backtracking procedure given below computes the orbit of $\beta[k]$ in the stabilizer of the points in $\beta[1 . . k-1]$. Due to the particular structure of the base $\beta$, this is exactly the operation of computing $\operatorname{orb}\left(f, \operatorname{stab}\left(F_{1}^{a}, G\right)\right)$ with $f=\min \left\{r \in F^{a}\right\}$ needed in Section 2 if we use $k=\left|F_{1}^{a}\right|+1$. It consists of an initializing procedure orbit_in_stabilizer() that calls a recursive procedure orb_in_stab().

orbit_in_stabilizer $(a, k)$

$/ *$ Returns the orbit of $\beta[k]$ in $\operatorname{stab}(\beta[1 . .(k-1)], G)$ where $G$ is the group represented by $T$ with base $\beta * /$

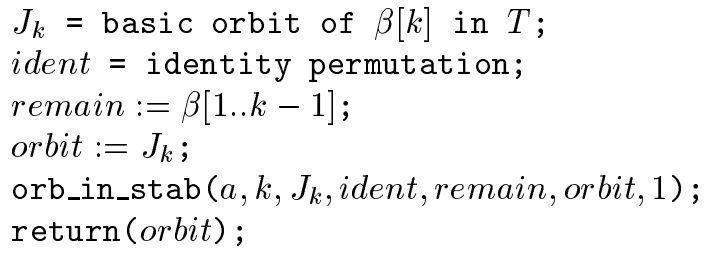

The parameters of the call to orb_in_stab() have the following interpretation: perm is a permutation in $G$ sending $\beta[1 .$. ind -1$]$ on a subset $B \subseteq \beta[1 . . k-1]$; remain is the set $\operatorname{perm}^{-1}(\beta[1 . . k-1]-B) ; J_{k}$ is the basic orbit of $\beta[k]$ in $T$; orbit 
is the set of points currently known in the orbit of $\beta[k]$ in $\operatorname{stab}(\beta[1 . .(k-1)], G)$; (orbit is passed by reference during the recursive calls;) ind refers to the point $\beta[i n d]$ being treated during the current call.

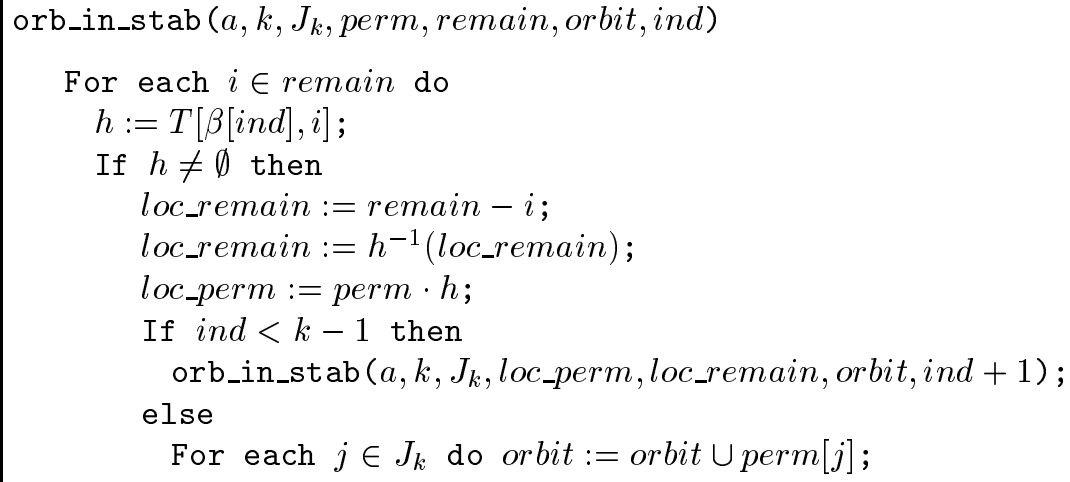

Proposition 3. The algorithm orbit_in_stabilizer() is correct.

Proof. Let $S=\beta[1 . .(k-1)]$. If $k=1, \operatorname{stab}(\emptyset, G)=G$ and the orbit of $\beta[1]$ in $G$ is $J_{1}$, as returned by the algorithm. Otherwise, we have $k \geq 2$. By Remark 3, $\operatorname{stab}(S, G)$ is generated by all permutations $g$ such that $g(S)=S$ with

$$
g=g_{1} \cdots \cdot g_{k-1} \cdot g_{k} \cdot h
$$

and $g_{i} \in U_{\beta[i]}$ for $i=1, \ldots k, h \in G_{k}$. Since $h[\beta[k]]=\beta[k]$,

$$
\begin{aligned}
& \operatorname{orb}(\beta[k], \operatorname{stab}(S, G))= \\
& \left\{v \in I^{n} \mid v=\left(g_{1} \cdots g_{k}\right)[\beta[k]], g_{i} \in U_{\beta[i]} \text { for } i=1, \ldots k, g(S)=S\right\} .
\end{aligned}
$$

Assume that $g_{i}=T\left[\beta[i], j_{i}\right]$ for $i=1, \ldots, k-1$. The condition $g(S)=S$ implies $j_{1} \in S$. Moreover, if $k \geq 3$ then $\left(g_{1} \cdot g_{2}\right)(\beta[2]) \in S-j_{1}$ and thus $g_{2}[\beta[2]] \in$ $g_{1}^{-1}\left(S-j_{1}\right)$. In general, for index $2 \leq$ ind $\leq k-1$, we have

$$
g_{\text {ind }}(\beta[i n d]) \in g_{\text {ind }-1}^{-1}\left(\ldots\left(g_{2}^{-1}\left(\left(g_{1}^{-1}\left(S-j_{1}\right)\right)-j_{2}\right)\right)-\cdots-j_{\text {ind }-1}\right) .
$$

Note that the set in (4) is exactly the parameter remain of the call to the procedure orb_in_stab() with value $i n d$ as last parameter. That procedure simply selects an index in this set, update perm and remain and calls itself recursively with ind +1 until ind $=k-1$ or no permutation $h$ is found. In the former case, $g_{1} \cdot \ldots \cdot g_{k-1}(S)=\operatorname{perm}(S)=S$, and it adds perm $[j]$ for all $j \in J_{k}$. This amounts to computing $\left(\right.$ perm $\left.\cdot g_{k}\right)[\beta[k]]$ for all $g_{k} \in U_{\beta[k]}$. In the latter case, the algorithm backtracks to ind - 1 , since no permutation in $G$ stabilizes $S$ with the current choice of permutations $g_{1}, \ldots, g_{\text {ind }-1}$. Since at each level in the recursion, all possible choices for $g_{\text {ind }}$ are explored, and the algorithm indeed returns the desired orbit. 
Remark 7. As observed in the justification above, the set in (4) is the current set remain. A weaker statement about this set is that remain $\subseteq \operatorname{perm}^{-1}(S)$, as

$$
\text { perm }^{-1}=g_{\text {ind }-1}^{-1} \cdots \cdots g_{2}^{-1} \cdot g_{1}^{-1} \text {. }
$$

Let us now turn to the question of deciding if set $S=\beta[1 . . k]$ is the lexicographically minimum set in $\operatorname{orb}(S, G)$. Note that for $k=\left|F_{1}^{a}\right|+1$, this is exactly the same question as deciding if $F_{1}^{a} \cup f$ is a representative, with $f=\min \left\{r \in F^{a}\right\}$ mentioned in Section 2. We assume that $\beta$ has the structure stated at the beginning of this section.

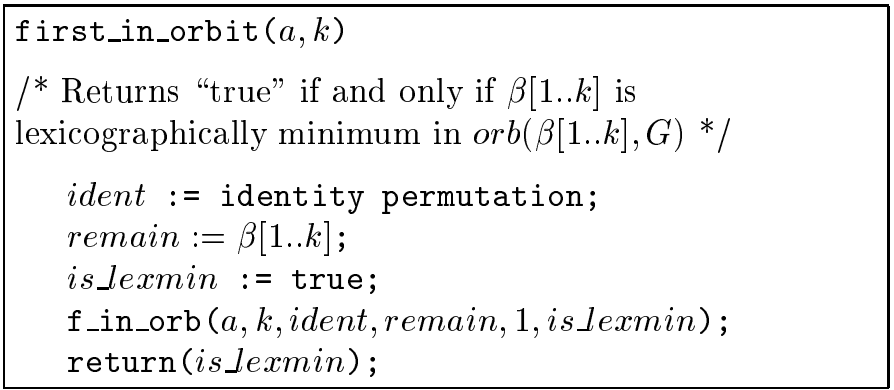

The parameters perm, remain and ind in the call to f_in_orb() are similar to the same parameters in the call of orb_in_stab(). The parameter is_lexmin is passed by reference and is used to stop the procedure as soon as it is known that $\beta[1 . . k]$ is not lexicographically minimum in $\operatorname{orb}(\beta[1 . . k], G)$.

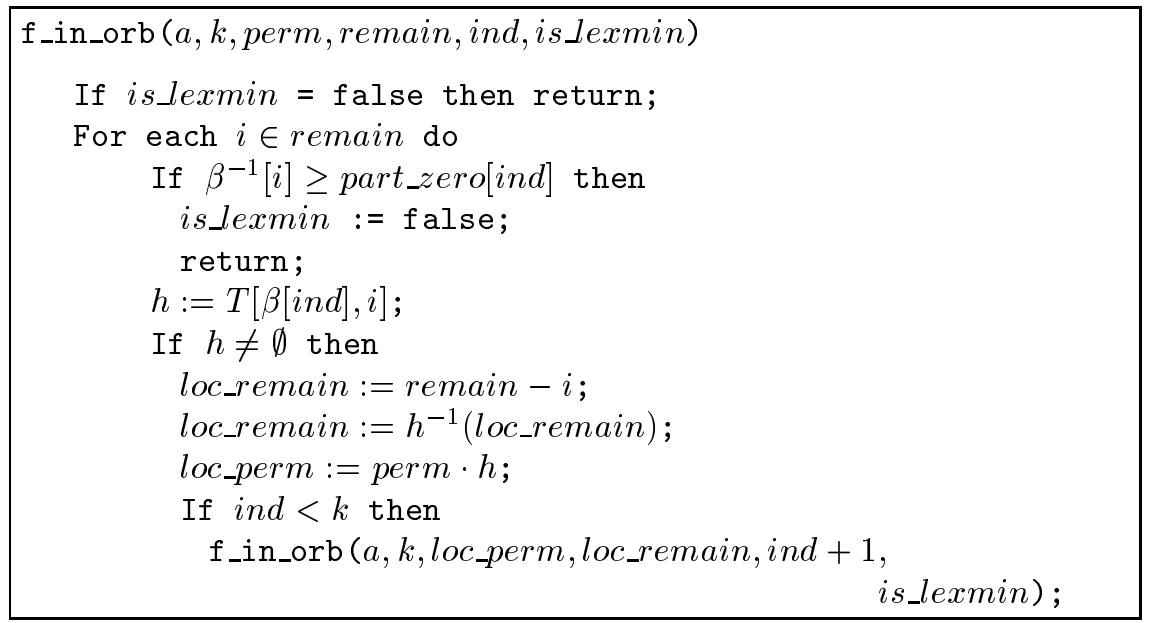


Proposition 4. The algorithm first_in_orbit() is correct.

Proof. Suppose that the condition

$$
\beta^{-1}[i] \geq \text { part_zero }[\text { ind }]
$$

in procedure f_in_orb() is satisfied. This condition means that, for some $t \leq i n d$, there exists a point $i$ in remain that has been fixed or set to 0 before fixing $\beta[t]$ to 1 and (if $t \geq 2$ ) after fixing $\beta[t-1]$ to 1 . Let

$$
S:=\operatorname{perm}(\beta[1 . . \text { ind }-1]) \subseteq \beta[1 . . k] \quad \text { i.e. } \quad \operatorname{perm}^{-1}(S)=\beta[1 . . \text { ind }-1] .
$$

Moreover, as pointed out in Remark 7 (the algorithms are similar, so this remark holds here too), remain $\subseteq \operatorname{perm}^{-1}(\beta[1 . . k])$ and since it is disjoint from $S$, we have

$$
i=\operatorname{perm}^{-1}[\beta[s]] \quad \text { for some } s \in\{\text { ind }, \ldots, k\} .
$$

Since $i$ was fixed or set to 0 before fixing $\beta[t]$ to 1 , we have, for some $w<\beta[t]$,

$$
i \in \operatorname{orb}(w, \operatorname{stab}(\beta[1 . . t-1], G)) .
$$

Hence there exists a permutation

$$
p \in \operatorname{stab}(\beta[1 . . t-1], G) \quad \text { with } \quad p(i)=w .
$$

Let $S^{\prime}:=\operatorname{perm}(\beta[1 . . t-1]) \subseteq S$. As $p(\beta[1 . . t-1])=\beta[1 . . t-1]$, we have

$$
\left(p \cdot \text { perm }^{-1}\right)\left(S^{\prime}\right)=\beta[1 . . t-1] \quad \text { and } \quad\left(p \cdot \text { perm }^{-1}\right)[\beta[s]]=w<\beta[t] .
$$

Thus $\left(p \cdot \operatorname{perm}^{-1}\right)\left(S^{\prime} \cup \beta[s]\right)=\beta[1 . . t-1] \cup w$ is lexicographically smaller than $\beta[1 . . t]$. It follows that when the algorithm returns "false", the set $\beta[1 . . k]$ is indeed not lexicographically minimal in its orbit under $G$.

Suppose now that the set $\beta[1 . . k]$ is not lexicographically minimal in its orbit under $G$. Let $p$ be a permutation such that $p(\beta[1 . . k])$ is lexicographically smaller than $\beta[1 . . k]$. Let $t$ be the smallest index in $\{1, \ldots k\}$ such that

$$
p(\beta[1 . . t-1])=\beta[1 . . t-1] \quad \text { and } \quad p(\beta[t])<\beta[t] .
$$

By Remark 3, we can write

$$
p=h_{1} \cdots h_{n}
$$

with $h_{j} \in U_{\beta[j]}$ for $j=1, \ldots, n$. Observe that $w=p(\beta[t])$ was fixed or set to 0 before fixing $\beta[t]$ to 1 . We have

$p^{-1}(\beta[1 . . t-1] \cup w)=\beta[1 . . t] \quad$ and thus $\quad p^{-1}[w]=\beta[s]$ for some $s \in\{1, \ldots, t\}$.

During the recursive calls to f_in_orb(), a permutation perm will occur with $\operatorname{perm}[\beta[i]]=p^{-1}[\beta[i]]$ for $i=1, \ldots, t-1$, namely

$$
\text { perm }=h_{1} \cdots h_{t-1} .
$$


Let $z:=\operatorname{perm}^{-1}[\beta[s]]$. Observe that

$$
\left(\operatorname{perm}^{-1} \cdot p^{-1}\right)[w]=z \quad \text { and } \quad \operatorname{perm}^{-1} \cdot p^{-1} \in \operatorname{stab}(\beta[1 . . t-1], G) .
$$

Hence $z \in \operatorname{orb}(w, \operatorname{stab}(\beta[1 . . t-1], G))$ and $z$ was fixed or set to 0 with $w$ (or earlier). It follows that remain contains $z$ and that $\beta^{-1}[z] \geq$ part_zero[t], implying that the algorithm will return "false".

Crude bounds on the worst case complexity of these two backtracking procedures are $O(n \cdot k !)$ and $O(n \cdot(k+1) !)$, respectively, but they turn out to be orders of magnitude faster on average, making them practical. (Values of $k$ in the range of 20 to 40 with $n \geq 200$ appear routinely in applications and are handled efficiently.)

Remark 8. For clarity, the algorithms orb_in_stab() and first_in_orbit() were presented separately, but it is possible to take advantage of their similarities to merge them into one single recursive procedure.

\section{Isomorphism Inequalities}

Let $a$ be a node of the enumeration tree and $H^{a}$ be the set of variables that are not fixed or set to 0 at node $a$. Suppose that there exists $J \subseteq H^{a}$ such that the representative $J^{*}$ of the orbit of $J$ under $G$ is lexicographically smaller than $F_{1}^{a}$. Then, if a node $b$ in the descendants of $a$ with $J \subseteq F_{1}^{b}$ exists, this node will be pruned by IP. Hence, the isomorphism inequality

$$
\sum_{j \in J} x_{j} \leq|J|-1
$$

is valid in the subtree rooted at $a$. Moreover, if the whole restricted enumeration tree is explored by a depth-first search, always selecting first the son $d$ where the branching variable is fixed to 1 , then the sets $F_{1}^{d}$ are enumerated in lexicographic order, starting with the smallest one. It follows that if an inequality (5) is generated at $a$, it is valid for the rest of the enumeration, i.e. it can be considered global.

The separation algorithm for the isomorphism inequalities is similar to the backtracking procedure for testing if a set is lexicographically minimal in its orbit under $G$. The parameters of the initializing procedure gen_iso_cuts() are simply the current node $a$, a subset $H \subseteq H^{a}$ (the motivation for using $H$ instead of $H^{a}$ will become clear later) and the current fractional solution $0 \leq \bar{x} \leq 1$. 


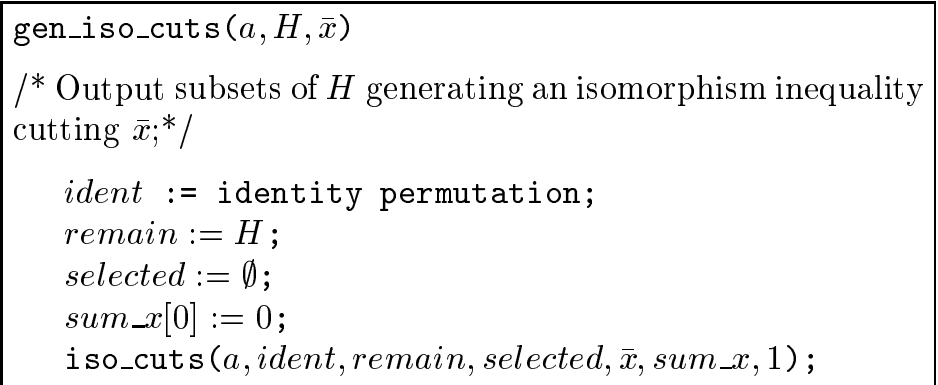

The parameters perm, remain and ind in the call to iso_cuts() are similar to the same parameters in the call of orb_in_stab(). The set selected is the ordered set of (ind - 1) points currently chosen; Finally, sum_ $x[k]$ gives the sum of the entries $\bar{x}_{i}$ for $i \in$ selected $[1, \ldots, k]$, for $k=1, \ldots$, ind -1 and sum_x $[0]=0$. Both selected and sum_x are passed by reference during the recursive calls.

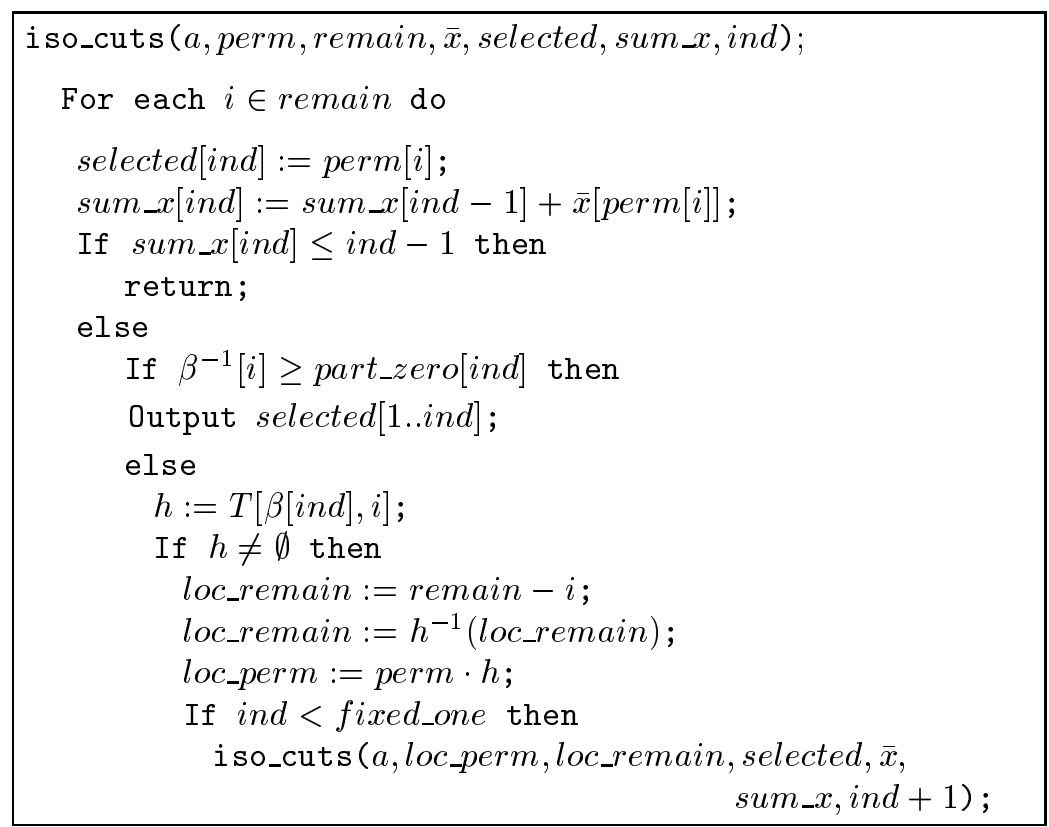

Proposition 5. The algorithm gen_iso_cuts() is correct.

Proof. Removing all tests and operations related to sum_x, this algorithm is similar to first_in_orbit() with $H$ replacing $\beta[1 . . k]$ for the initialization of remain. The proof that each set in the output is indeed a set whose representative is lexicographically smaller than $F_{1}^{a}$ is almost identical to the similar proof for 
first_in_orbit(). The proof that all minimal sets generating an isomorphism inequality are in the output is also similar to the proof of Proposition 4.

The operations related to sum $\_x$ simply update its entries so that $s u m \_x[$ ind $]$ is the sum of the entries $\bar{x}_{i}$ for $i \in$ selected $[1, \ldots, i n d]$. The condition "If sum_x $[$ ind $] \leq$ ind $-1 \ldots$..." in iso_cuts() is used to identify sets that cannot be extended to a set generating an isomorphism inequality cutting $\bar{x}$. When the condition is not met, the algorithm backtracks to the recursive call with parameter ind -1 .

Crude estimates for the worst case complexity of gen_iso_cuts() is in $O(n \cdot|H| !)$ but, in practice, it is able to handle efficiently instances with $|H| \geq 100$ and $n \geq 200$.

It is now time to discuss the way to pick the set $H \subseteq H^{a}$. If $H=H^{a}$ then the above algorithm is an exact separation algorithm. The purpose of selecting a set $H$ smaller than $H^{a}$ is to get a heuristic separation procedure faster than the exact algorithm. Note that including in $H$ an index $i$ with $\bar{x}_{i}=0$ is pointless, and that finding a subset $J$ with $\bar{x}(J)>|J|-1$ is more probable when $J$ is a subset of the indices $i$ with $\bar{x}_{i}$ relatively large. Thus, a sensible choice is to set $H$ as all indices $i$ such that $\bar{x}_{i}>\delta$ for some $\delta>0$.

Three additional remarks on this algorithm: first, the use of a vector sum_x[k] instead of a single variable, say sum_x, avoids the update of sum_x when performing a backtracking step. This prevents rounding errors propagating during the course of the algorithm, as entries in $\bar{x}$ are rational numbers. Second, it may happen that the same set $J$ appears several times in the output of the algorithm, since different ordering of its elements may yield a set with representative better than $F_{1}^{a}$. Finally, non-minimal sets can also be in the output. Since, if $J_{1} \subseteq J_{2}$, the isomorphism cut generated by $J_{1}$ implies the one generated by $J_{2}$, removing duplicates and non-minimal sets in the output is advisable.

\section{Applications}

We use the software ABACUS (version 2.3) developed by Thienel [10], [19], [37], now distributed by OREAS [32], as generic implementation of all branch-and-cut steps (isomorphism pruning excepted), and the LP solver is CPLEX7.1 [9]. We briefly describe results obtained on three applications: covering designs, error correcting codes and hard covering problems. Files of the test problems (in LP format) can be obtained from [28].

Let $V$ be a set of elements of cardinality $v$ and let $k$ and $t$ be integers such that $v \geq k \geq t \geq 0$. Let $\mathcal{K}$ be the set of all $k$-subsets of $V$ and $\mathcal{T}$ be the set of all $t$-subsets of $V$. A $(v, k, t)$-covering design is a collection $\mathcal{C}$ of sets in $\mathcal{K}$ such that 
each $t \in \mathcal{T}$ is contained in at least one set of $\mathcal{C}$. A $(v, k, t)$-covering design $\mathcal{C}$ is minimum if the cardinality of $\mathcal{C}$ is as small as possible.

Covering designs have a long history and have applications in statistics, coding theory and combinatorics, among others. Numerous theorems give the value of a minimum covering design under certain assumptions on the parameters (see the survey [30]). Yet, for particular values of the parameters, only lower and upper bounds are available. A case in point is the $(10,5,4)$-covering design, for which a lower bound of 50 and an upper bound of 51 are known [11].

Running the described branch-and-cut algorithm for the (10,5,4)-covering design problem, while pruning nodes as soon as their associated LP relaxation has value strictly larger than 50 , we obtain a proof that no solution better than the best known solution of 51 exists (see [27] for the ILP formulation and details). The ILP (cov1054.lp) has 252 variables, 384 inequalities and the symmetry group $G$ has order $10 !=3,628,800$. The average number of non-empty entries in the Schreier-Sims table over all nodes of branch-and-cut is about 550. There are only 335 nodes in the enumeration tree and the cpu time (in seconds) is distributed as follows (the machine used is an HP B2000 running HP-UX11 with a 500MHz PA-8600 CPU): Total cpu time: 82.83 , LP cpu time: 72.09 , Pool separation for inactive inequalities: 0.13 , Separation for isomorphism inequalities: 2.50, Operations related to the symmetry group: 9.57 .

Although the separation for isomorphism inequalities might seem time consuming, this should be balanced with the fact that not using these inequalities makes the branch-and-cut enumeration tree grow from 335 nodes to 495 . (These numbers and running times are slightly better than those in [27] where no 0-setting and a less general isomorphism pruning were used). It is worth noting that proving that this ILP has no solution with value 50 is not possible for the branch-andcut of CPLEX7.1. Even adding straightforward symmetry breaking inequalities to the ILP formulation does not help much: adding the constraints requiring that the number of chosen sets containing element $i$ is larger than the number of chosen sets containing the element $i+1$, for $i=1, \ldots, 9$ and setting $x_{0}$ to 1 yields an ILP (cov1054sb.lp) still difficult for CPLEX7.1. Using an upper bound cutoff of 50.0001, CPLEX7.1 is far from done after more than 60 hours CPU and 3.5 million nodes (with about 300,000 of them still unfathomed).

An error correcting binary code with distance $d$ and word length $w$ is a collection $\mathcal{C}$ of binary $w$-vectors such that the Hamming distance between any pair of vectors in $\mathcal{C}$ is at least $d$ (Chapter 9 in [8]). The maximum number of vectors in $\mathcal{C}$ is denoted by $A(w, d)$. Here also, for small values of $w$ and $d$, only bounds on $A(w, d)$ are known. For example, $72 \leq A(10,3) \leq 76$ [25]. A simple set packing formulation with one variable per binary $w$-vector with at least three 1's yields an ILP with a group of order $w$ !. This ILP for finding $A(8,3)$ $(\operatorname{cod} 83 r . l p)$ is difficult for the branch-and-cut of CPLEX7.1 as more than 6 hours and about 1 million nodes are needed to solve the problem. The isomorphism pruning algorithm described in this paper, however, does it in 143 nodes and 
9 seconds CPU. The ILP has 219 variables, 219 inequalities and the symmetry group $G$ has order $8 !=40,320$. The average number of non-empty entries in the Schreier-Sims table over all nodes of the branch-and-cut is about 312 . The cpu time (in seconds) is distributed as follows: Total cpu time: 8.52, LP cpu time: 6.52, Pool separation for inactive inequalities: 0.03, Separation for isomorphism inequalities: 0.05 , Operations related to the symmetry group: 1.05 .

Fulkerson [13] introduced a class of difficult set covering problems obtained from the incidence matrix of Steiner triple systems [15]. As an indication on the difficulty of these problems, Avis [1] showed that any branch-and-bound algorithm using LP relaxations and dominance pruning will enumerate at least $2^{\sqrt{2 n / 3}}$ nodes for an infinite family of such problems on $n$ variables with $n \rightarrow \infty$. This class of problems is a good example of problems with huge symmetry groups, but for which finding symmetry breaking inequalities is not easy. Feo and Resende [12] studied similar problems called STS81 and STS243, and found good heuristic solutions, but only a few years ago Mannino and Sassano [26] were able to solve STS 81 to optimality. Their branch-and-bound requires an enumeration tree with more than 900 million nodes. We report results for the problem known as $S T S 81$ (sts $81 . l p)$. The symmetry group was computed using the program nauty (version 1.5) written by McKay [31]. CPLEX7.1 is not able to prove optimality of the optimal value of STS81.

The isomorphism pruning algorithm described in this paper, however, does it in 385 nodes and 161.95 seconds CPU. The ILP has 81 variables, 1080 inequalities and the symmetry group $G$ has order $1,965,150,720$. The average number of non-empty entries in the Schreier-Sims table over all nodes of the branch-andcut is about 442. The cpu time (in seconds) is distributed as follows: Total cpu time: 161.95, LP cpu time: 22.38, Pool separation for inactive inequalities: 1.92, Separation for isomorphism inequalities: 118.80 , Operations related to the symmetry group: 14.69 .

It is of course shocking to spend $75 \%$ of the time for the generation of isomorphism inequalities. Two main reasons can explain this: first, the order of the symmetry group is much larger than in other applications; second, the set $H$ used by default by the algorithm tends to be relatively large, slowing down the procedure. Tuning the parameters for the generation of the inequalities (choice of $H$, frequency of the generation) could improve the results significantly. For example, turning off the generation of isomorphism cuts yields an enumeration tree of 659 nodes with the following statistics: Total cpu time: $64.04, \mathrm{LP}$ cpu time: 37.92 , Pool separation for inactive inequalities: 0.03 , Operations related to the symmetry group: 19.83 .

Acknowledgements. I wish to thank two anonymous referees for their detailed comments and for correcting a mistake in an earlier version of the proof of Proposition 4.3. 


\section{References}

1. Avis D., "A Note on Some Computationally Difficult Set Covering Problems", Mathematical Programming 8 (1980), 138-145.

2. Babai L., Luks E.M., Seress Á., "Fast Management of Permutation Groups I", SIAM Journal on Computing 26 (1997), 1310-1342.

3. Brown C.A., Finkelstein L., Purdom P.W., "A New Base Change Algorithm for Permutation Groups", SIAM Journal on Computing 18, (1989), 1037-1047.

4. Butler G. "Computing in Permutation and Matrix Groups II: Backtrack Algorithm", Mathematics of Computation 39 (1982), 671-680.

5. Butler G., Fundamental Algorithms for Permutation Groups, Lecture Notes in Computer Science 559, Springer (1991).

6. Butler G., Cannon J.J., "Computing in Permutation and Matrix Groups I: Normal Closure, Commutator Subgroups, Series", Mathematics of Computation 39 (1982), 663-670.

7. Butler G., Lam W.H., "A General Backtrack Algorithm for the Isomorphism Problem of Combinatorial Objects", J. Symbolic Computation 1 (1985), 363-381.

8. Conway J.H., Sloane N.J.A., Sphere Packings, Lattices and Groups, Springer (1993).

9. ILOG CPLEX 7.1 User's Manual, (2001).

10. Elf M., Gutwenger C., Jünger M., Rinaldi G., "Branch-and-Cut Algorithms for Combinatorial Optimization and their Implementation in ABACUS", in Jünger M., Naddef D., eds., Lecture Notes in Computer Science 2241, Springer (2001), 155-222.

11. Etzion T., Wei V., Zhang Z., "Bounds on the Sizes of Constant Weight Covering Codes", Designs, Codes and Cryptography 5 (1995), 217-239.

12. Feo T.A., Resende G.C., "A Probabilistic Heuristic for a Computationally Difficult Set Covering Problem", Operations Research Letters 8 (1989), 67-71.

13. Fulkerson D.R., Nemhauser G.L., Trotter L.E., "Two Computationally difficult Set Covering Problems That Arise in Computing the 1-width of Incidence Matrices of Steiner Triple Systems", Mathematical Programming Study 2, (1974), 72-81.

14. Gibbons P.B., "Computational Methods in Design Theory", in: The CRC Handbook of Combinatorial Designs, Colbourn C.J., Dinitz J.H., eds., CRC Press (1996), 718-740.

15. Hall M., Combinatorial Theory, Wiley (1986).

16. Hoffman C.M., Group-Theoretic Algorithms and Graph Isomorphism, Lecture Notes in Computer Science 136, Springer (1982).

17. Ivanov A.V., "Constructive Enumeration of Incidence Systems", Annals of Discrete Mathematics 26 (1985), 227-246.

18. Jerrum M., "A Compact Representation for Permutation Groups", Journal of Algorithms 7 (1986), 60-78.

19. Jünger M., Thienel S., "Introduction to ABACUS - A Branch-And-CUt System", Operations Research Letters 22 (1998), 83-95.

20. Kreher D.L., Stinson D.R., Combinatorial Algorithms, Generation, Enumeration, and Search, CRC Press (1999).

21. Leon J.S., "On an Algorithm for Finding a Base and a Strong Generating Set for a Group Given by Generating Permutations", Mathematics of Computation 35 (1980), 941-974.

22. Leon J.S., "Computing Automorphism Groups of Combinatorial Objects", in Computational Group Theory, Atkinson M.D. (ed.), Academic Press (1984), 321-335.

23. Luetolf C., Margot F., "A Catalog of Minimally Nonideal Matrices", Mathematical Methods of Operations Research 47 (1998), 221-241.

24. Luks E., "Permutation Groups and Polynomial-Time Computation", in DIMACS Series in Discrete Mathematics and Theoretical Computer Science 11 (1993), Groups and Computation, L. Finkelsein, W. Kantor, eds., 139-175.

25. S. Lytsin, "An Updated Table of the Best Binary Codes Known", in Handbook of Coding Theory, V.S. Pless, W.C. Huffmann, eds., North-Holland, Elsevier (1998).

26. Mannino C., Sassano A., "Solving Hard Set Covering Problems", Operations Research Letters 18 (1995), 1-5.

27. Margot F., "Small Covering Designs by Branch-and-Cut", To appear in Mathematical Programming.

28. http://www.ms . uky. edu/ fmargot

29. McKay D., "Isomorph-free Exhaustive Generation", Journal of Algorithms 26 (1998), 306324.

30. Mills W.H., Mullin R.C., "Coverings and Packings", in: Contemporary Design Theory: A collection of Surveys, Dinitz J.H., Stinson D.R., eds., Wiley (1992), 371-399. 
31. McKay B.D., "Nauty User's Guide (Version 1.5)", Computer Science Department, Australian National University, Canberra.

32. http://www.oreas.de

33. M.W. Padberg, G. Rinaldi, "A Branch-and-Cut Algorithm for the Resolution of Large Scale Symmetric Travelling Salesman Problems", SIAM Review 33 (1991), 60-100.

34. Read R.C., "Every one a winner or how to avoid isomorphism search when cataloguing combinatorial configurations", Annals of Discrete Mathematics 2 (1978), 107-120.

35. Seah E., Stinson D.R., "An Enumeration of Non-isomorphic One-facto-rizations and Howell Designs for the Graph $K_{10}$ minus a One-factor", Ars Combinatorica 21 (1986), 145-161.

36. Seress Á., "Nearly Linear Time Algorithms for Permutation Groups: An Interplay Between Theory and Practice", Acta Applicandae Mathematicae 52 (1998), 183-207.

37. Thienel S., "ABACUS - A Branch-And-CUt System" Ph.D. Thesis, Universität zu Köln (1995).

38. L.A. Wolsey, Integer Programming, Wiley (1998). 